\title{
Numerical Simulation of Two-Dimensional Large-Amplitude Acoustic Oscillations
}

\author{
Heying Feng ${ }^{1 *}$, Yehui Peng ${ }^{2}$, Jianshi Gong ${ }^{1}$ and Fenglin Yin ${ }^{2}$ \\ ${ }^{* 1}$ Hunan Provincial Key Laboratory of Health Maintenance for Mechanical Equipment, Hunan \\ University of Science and Technology, Xiangtan 411201, People's Republic of China \\ ${ }^{2}$ School of Mathematics and Computational Science, Hunan University of Science and \\ Technology, Xiangtan 411201, People's Republic of China
}

Email: fengheying@hnust.edu.cn

\begin{abstract}
The two-dimensional nonlinear acoustic oscillations in the resonator of four different driving amplitudes are simulated by a gas-kinetic scheme. The shock in the resonator, the effects of the driving amplitude on the acoustic field and flow and the distribution of harmonics are investigated based on the simulaton results. Moreover, this work discusses the reasons for the nonlinear effects such as annular effect and velocity reverse. It is pointed out that the waveforms of the acoustics variables increasingly distort with the driving-amplitude increasing. And it is found that the acoustic field and the flow under the large-amplitude are quite different from those under the finite-amplitude. Moreover, it has been shown that the shock greatly affects the pressure, temperature and the axial velocity and has no effect on the radial velocity. All of this shows that the largeamplitude has a large effect on the nonlinearity in the resonator.
\end{abstract}

Keywords: Two-dimensional flow field, Gas-kinetic scheme, Large-amplitude oscillation, Nonlinear effect.

\section{INTRODUCTION}

The finite or large amplitude acoustic oscillations can improve the power density and conversion efficiency of thermo-acoustic devices, and can produce a larger pressure ratio for some acoustics devices. Therefore, there are many applications for the finite or large amplitude acoustic oscillations in practical thermo-acoustics and acoustics devices. However, there are many nonlinear dissipations accompanied by large amplitude oscillation, which is a critical issue in the application of thermo-acoustics. Hence, it is very important to recognize the acoustic field characteristics and the nonlinear dissipative mechanism in the resonator.

Currently, there are many investigations on the nonlinear acoustic oscillations in the resonator, many of which are based on the one-dimensional model [1]-[6]. There is an urgent need for a two-dimensional model of large amplitude acoustic oscillation. Vanhille etc. presented a twodimensional model [7] for the cylinder resonator, they presented a finite volume scheme based on this model to simulate the nonlinear oscillations and analyze the pressure distribution in the resonator [8]. Additionally, Vanhille etc. [9]-[10] has proposed a numerical model for the nonlinear stand wave by expanding the state equation to the second order. This model includes the continuity equation, the momentum equations and the energy equation. In the model, the momentum equations have no limits to the nonlinearly.
However, the continuity equation is only suitable in the case of finite amplitude and the energy equation only comprises the viscosity without the heat conduction. Under the consideration of irrotationality, Cervenka etc. [11] investigated the nonlinear two-dimensional standing wave in the resonator. Alexeev etc. [12] investigated the nonlinear gas oscillations and acoustic field in the resonator by solving the Navier-Stokes equations though using the finite difference method and verified the simulation results by experiments. Their results showed that the temperature distribution is related to the nonlinearity of flow with periodic shocks and to the turbulence intensity along the tube. Tang etc. [13] solved the Navier-Stokes equations by the KFVS (Kinetic Flux Vector Splitting) method to simulate the nonlinear oscillations in the two-dimensional resonator. But they did not present the two-dimensional flow field. Wang etc. [14] presented a lattice Boltzmann model for the two-dimensional oscillations in the resonator. By taking the periodic boundary condition, they simulated a few acoustic fields driven by five different frequencies related to the resonant frequency. However, their two-dimensional model only showed some one-dimensional characteristics. Ning etc. [15] developed a two-dimension model for the axial symmetry resonator. They resolved the model by the finite volume method and obtained the pressure distribution without the flow field. Aganin et al [16] made a comparison of solutions of the gas oscillation in a closed tube for two ideal gas models, the entropy conservation model and the total energy model, with finite 
difference method. They found that one can obtain steady solutions rapidly by making use of the entropy conservation model for high-amplitude gas oscillation. However, the influence of entropy changes in the course of development of longitudinal gas oscillations in the tube is considered in their studies only in one dimension.

The gas-kinetic BGK scheme (GKS-BGK) [17] has some advantages over high-dimensional and large-Mach compressible fluid and nonlinear simulation, for the NavierStokes equations can be exactly recovered from the BGK model due to the connection between the viscosity coefficient and the relaxation time. The results in Refs. [18], [19] and [20] showed that the GKS has competitive accuracy and efficiency in simulating the two-dimensional acoustic oscillations. In particular, ref. [20] showed that the GKS has the capability to simulate the nonlinear effects in the resonator, such as large-amplitude oscillations, eddies, acoustics streaming, etc. On the foundation of ref. [20], this work investigated the characteristics of the nonlinear acoustics flow field in the resonators driven by the four different amplitudes varying from finite-amplitude to largeamplitude, and placed an emphasis on analyzing the mechanism and effects of these nonlinear phenomena, and numerically analyzed some nonlinear dissipations in the resonator. The effect of large-amplitude on the nonlinearity in the resonator is investigated in particular. All of these are beneficial to the optimal design of resonators.

\section{GAS-KINETIC BGK SCHEME}

The present gas-kinetic BGK (GKS-BGK) scheme is based on the two-dimensional BGK-Boltzmann equation

$f_{t}+u f_{x}+v f_{y}=(g-f) / \tau$

where $f$ is the gas distribution function and $g$ is the equilibrium state approached by $f$. Here, $f$ and $g$ are functions of time $t$, space $(x, y)$, particle velocities $(u, v)$ and the internal variable $\xi$, and $\tau$ is the particle collision time. Usually, the equilibrium state $g$ is assumed as a Maxwellian distribution

$$
g=\rho(\lambda / \pi)^{(\mathrm{K}+2) / 2} \exp \left[-\lambda\left((u-U)^{2}+(v-V)^{2}+\xi^{2}\right)\right]
$$

where $\rho$ is the density, $U$ and $V$ are the macroscopic velocity in the $x$ - and $y$-direictions respectively, and $\lambda=1 / 2 R T$ is the function of temperature $T$. The internal variable $\xi^{2}$ is equal to $\xi^{2}=\xi_{1}^{2}+\xi_{2}^{2}+\cdots+\xi_{K}^{2}$, where the total number of degrees of freedom $K=(5-3 \gamma) /(\gamma-1)+1$ for the 2D gas flow and $\gamma$ is the ratio of the principal specific heat. Since mass, momentum, and energy are conserved during particle collisions, $f$ and $g$ satisfy the conservation constraint

$$
\int(g-f) \psi d \Xi=0
$$

where $d \Xi=d u d v d \xi$ and

$$
\boldsymbol{\psi}=\left(\psi_{1}, \psi_{2}, \psi_{3}, \psi_{4}\right)^{\mathrm{T}}=\left(1, u, v,\left(u^{2}+v^{2}+\xi^{2}\right) / 2\right)^{\mathrm{T}}
$$

Taking moments $\boldsymbol{\psi}$ in Eq. (1) and integrating it with respect to $d \Xi$ in phase space, $d S$ in a numerical cell $S_{i, j}$, and $d t$ in a time step $\left[t^{n}, t^{n+1}\right]$, yields

$\mathbf{W}_{i, j}^{n+1}=\mathbf{W}_{i, j}^{n}+\int_{\partial \Omega_{i, j}} \int_{t^{n}}^{t^{n+1}} \mathbf{F} d t d l /\left|S_{i, j}\right|$

where $\mathbf{W}_{i, j}^{n+1}$ and $\mathbf{W}_{i, j}^{n}$ are the cell average macroscopic conservative quantities over the numerical cell $S_{i, j}$ with boundary $\partial \Omega_{i, j}$, and area $\left|S_{i, j}\right|$ at time $t^{n+1}$ and $t^{n}$, respectively. The macroscopic values $\mathbf{W}$ and the flux $\mathbf{F}$ can be computed from the gas distribution $f$ as follows:

$\mathbf{W}=(\rho, \rho U, \rho V, \rho e)^{\mathrm{T}}=\int f \psi d \Xi, \quad \mathbf{F}=\int u f \psi d \Xi$

where $\rho e=p /(\gamma-1)+\rho\left(U^{2}+V^{2}\right) / 2$.

Eqs. (5) and (6) imply that it is crucial for the GKS-BGK to get the distribution function $f$ at the cell interface. The above numerical scheme is second-order ${ }^{[18]}$. Interested readers can refer to refs. [17] and [18] for more details about GKS.

\section{PHYSICAL MODEL}

Fig. 1 shows the physical object of this study. It is a gasfilled cylindrical tube closed at one end, and driven by a piston at the other end. The adiabatic and non-slip boundary conditions are imposed on the wall except for at the driving end. The driving frequency is set at $f=666 \mathrm{HZ}$, corresponding to the wave length $\lambda=51.6 \mathrm{~cm}$ under the ambient temperature $298 \mathrm{~K}$. The length of the resonator $L$ is set at half of the wave length, that is $L=\lambda / 2=25.8 \mathrm{~cm}$. The diameter of the resonator is $H=0.15 L=3.87 \mathrm{~cm}$. The velocity of the piston is set at $u_{0}=l \omega \sin \omega t$, where $l$ is the amplitude of the displacement of the piston, $\omega=2 \pi f$ is the angular frequency and $l \omega$ is the amplitude of the velocity of the piston.

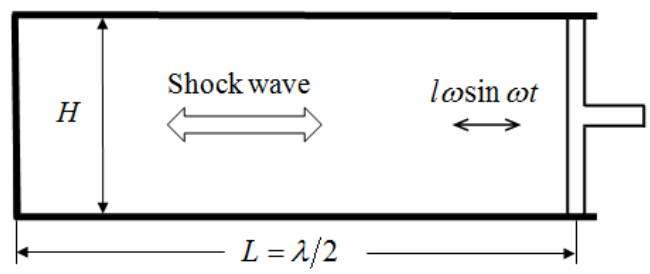

Figure 1. Schematic description of the resonator driven by the piston

In the simulation, the full compressible two-dimensional Navier-Stokes equation is chosen to model the acoustic field and the flow field which is produced by the interaction of the acoustic wave and the wall. The computation region $L \times H$ is uniformly divided into $400 \times 200$ grids. Initially, the density, 
pressure and temperature are all uniformly distributed. Moreover, the initial density, pressure and kinetic viscosity are set at $\rho_{0}=1.29 \mathrm{~kg} / \mathrm{m}^{3}, p_{0}=1.1033 \times 10^{5} \mathrm{~Pa}$ and $v=1.56 \times 10^{-5} \mathrm{~m}^{2} / \mathrm{s}$, respectively. In the computation, the four different amplitudes of the piston displacement are chosen to cover finite-amplitude and large-amplitude cases. In this choice, the order of the non-dimensional displacement of the oscillation varies from $10^{-4}$ to $10^{-3}$ and the order of the Mach number varies from $10^{-4}$ to $10^{-2}$.

Table 1. Four Computation Cases

\begin{tabular}{ccccc}
\hline & $\begin{array}{c}l \\
(\mu \mathrm{m})\end{array}$ & $l / L$ & $M a=l \omega / c_{0}$ & $\varepsilon=\sqrt{\pi l / L}$ \\
\hline Case 1 & 60 & $2.33 \times 10^{-4}$ & $7.30 \times 10^{-4}$ & 0.027 \\
Case 2 & 120 & $4.65 \times 10^{-4}$ & $1.46 \times 10^{-3}$ & 0.038 \\
Case 3 & 510 & $1.97 \times 10^{-3}$ & $6.20 \times 10^{-3}$ & 0.079 \\
Case 4 & 1040 & $4.03 \times 10^{-3}$ & $1.27 \times 10^{-2}$ & 0.113 \\
\hline
\end{tabular}

\section{NUMERICAL RESULTS AND DISCUSSION}

Firstly, the numerical convergence with the grid number increasing is examined on three kinds of grids, $128 \times 16$, $256 \times 32$ and $512 \times 64$, which are numerically tested for 20 periods. Fig. 2 shows the convergence with grid number, where it can be seen that these results with different grids are almost the same except for a slight difference near the valley of the curves. Therefore, the grid of $256 \times 32$ is sufficient for the problems under consideration and also provides good resolution of the discepancies. Hence the grid $256 \times 32$ is used in the following computation.

Fig. 3 gives a comparison of the pressure oscillation form, which is calculated at the closed end $(x / L=1.0)$ for resonant frequency, with the results from Wang et al.'s numerical results [14], Aganin et al.'s numerical results of model A [16] , Chester's theoretical results [6] and the experimental data of Saenger and Hudson [20]. Fig. 3(a) shows a good agreement between our numerical results with previous numerical results and theory results. The plot also shows that the pressure waveform in our study is more asymmetric with regard to the static pressure than Chester's results. This is because the viscosity and heat conduction were taken into account in our study, energy transfer to second even higher harmonics with nonlinearity. Moreover, the numerical pressure waveform in our study is similar with that in the experiment results of Saenger and Hudson [20]. The good agreement confirms the accuracy of the method and relevant code.

In the following computations, the verified kinetic scheme was used for simulation of two-dimension high-amplitude acoustic oscillation in the resonator.

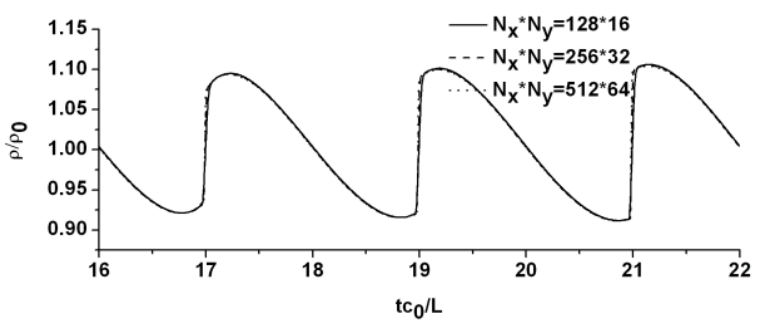

(a)
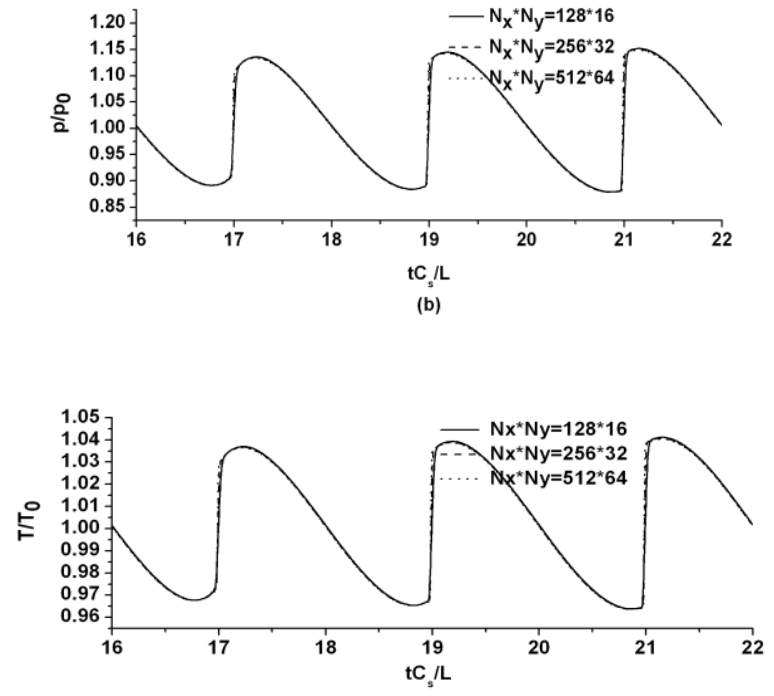

(c)

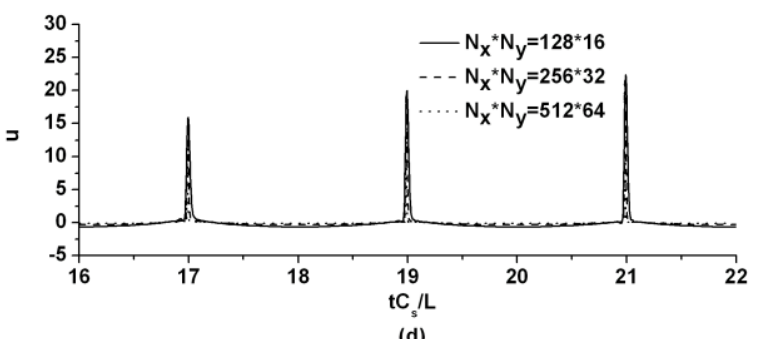

Figure 2. Mesh refinement study of the time dependency (a) density; (b) pressure; (c) temperature; (d) longitudinal velocity at $x / L=0.998$ and $y / D=0.484$ with the adiabatic boundary condition for resonant frequency

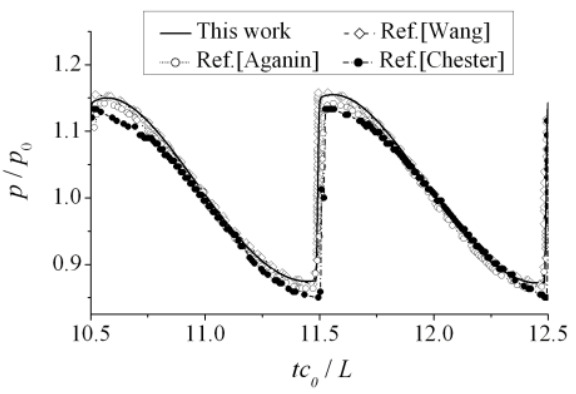

(a)

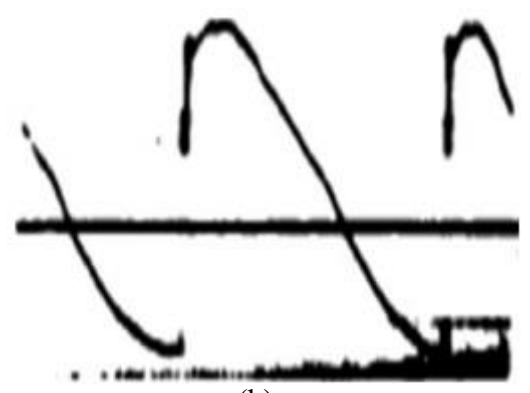

(b)

Figure 3. (a) Comparison of the numerical results in this study ( - ) with Wang et al.'s results [14](- - - ), Aganin et al.'s results [16] (....), and Chester's results [6] (......). (b) The experimental results of Saenger and Hudson[20]. 
Figs. 4-7 show the distributions of relative pressure, relative temperature, axial velocity and radial velocity in the resonator driven by four different amplitudes at the nondimensional moment $t c_{0} / 2 L=80$, respectively. In the figures, the $x$-direction is the flow direction, the y-direction is the cross section direction, the $z$-direction is the magnitude of the quantity. Figs.4-7 have several implications: 1) With an increase in the driving amplitude, the amplitudes of the oscillation wave of the pressure, temperature, axial velocity and radial velocity all become larger, the wave form of these quantities become more complicated, the phases are changed, and the position of the shock wave is also changed. However, the positions of the shock wave for pressure, temperature and velocity are in agreement with each other for the same driving amplitude. 2) Generally, with an increase in the driving amplitude, the temperature and the axial velocity transform from the quasi one-dimension to the two-dimensions (see Figs.5-6) and the radial velocity wave transforms from regular to irregular (see Fig.7). Moreover, the ratio $\mid v_{\max } /$ $u_{\max } \mid$ of the magnitude of radial velocity to that of axial velocity is greatly affected by the driving amplitude. The ration is $0.35 \% \sim 10^{-3}$ for $l=60 \mu \mathrm{m}$ while it is $3.6 \% \sim 10^{-2}$ for $l=1040 \mu \mathrm{m}$, which implies that the radial velocity might be neglected for the finite amplitude compared to the axial velocity while it is comparable with the axial velocity for the large amplitude. However, Fig.4 implies that the pressure is only the function of $\mathrm{x}$, which conforms to the usual assumption $d p / d y=0.3)$ Fig.5 shows that the temperature in the vicinity $(y / H \approx 0$ and 1$)$ of the wall is higher than the temperature in the center region whether at the wave front or after the wave in the cases of large-amplitude. That is, there is a circulatory pattern in large-amplitude cases whether at the wave front or after the wave. However, the temperature circulatory pattern occurs only at the wave front in finiteamplitude cases, for the effect disappears after the shock wave. 4) Similarly, one finds from Fig.6 (c) and (d) that there are velocity circulatory patterns in large-amplitude cases, whether at the wave front or after the wave, since the magnitude of the axial velocity in the vicinity of the wall is larger than that at the center region. Additionally, the velocity is changed dramatically at the position where the shock wave occurs in large-amplitude cases. The velocity in the center region is changed from negative maximum to zero, while the velocity in the vicinity of the wall is changed from negative maximum to positive velocity. That is, the gradient of the velocity in the vicinity of the wall is greater than that at the center. Moreover, there is reverse velocity after the shock wave in the vicinity of the wall. All of these phenomena are not evident in the cases of finite-amplitude (see Figs. 6 (a) and 6 (b)). 5) The temperature circulatory pattern and reverse velocity become more and more evident with an increase in the driving amplitude, which implies that the one- or quasione-dimensional model is not suitable to model the nonlinearity in the flow, heat exchange and acoustic transmission in the large-amplitude resonator. 6) The shock wave has a great effect on the pressure, temperature and axial velocity, but it has little affect on the radial velocity. Figs. (4)-(6) show a great gradient of pressure, temperature and axial velocity at the shock wave position. Fig. (7) implies there is no shock wave in the radial velocity.

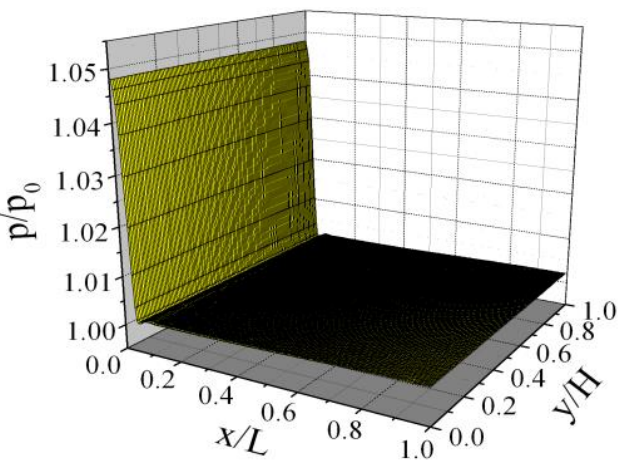

(a)

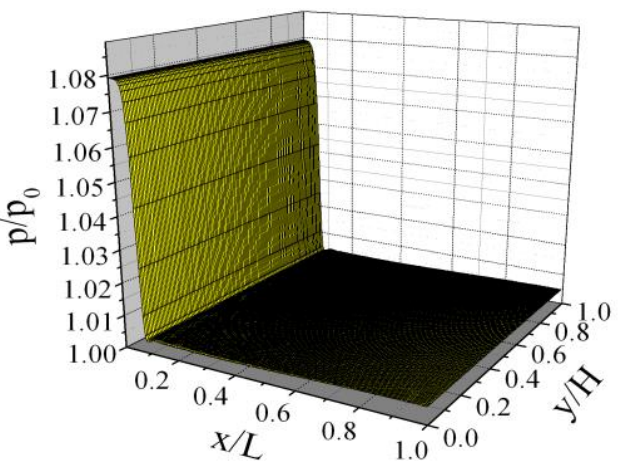

(b)

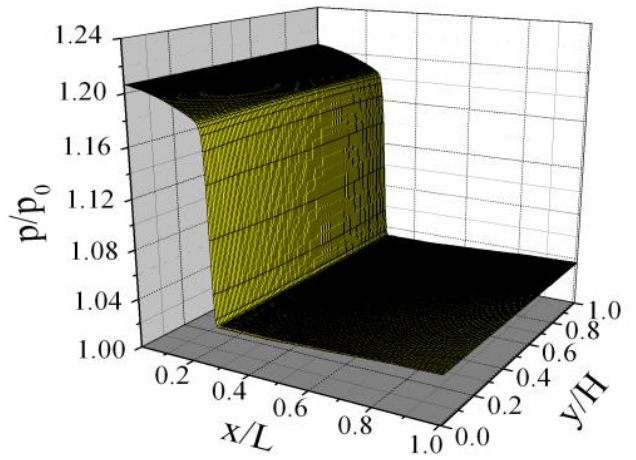

(c)

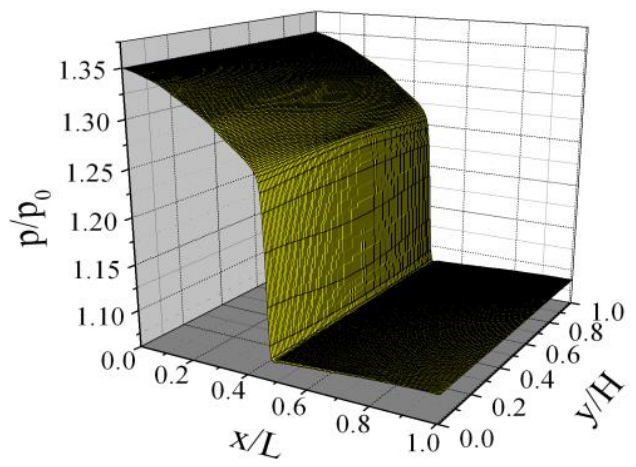

(d)

Figure 4. Instantaneous pressure distributions for four different driving amplitudes at $t c_{0} / 2 L=80$ with the condition of adiabatic boundary for resonant frequency.(a) $l=60 \mu \mathrm{m}$; (b) $l=120 \mu \mathrm{m}$; (c) $l=510 \mu \mathrm{m}$; (d) $l=1040 \mu \mathrm{m}$ 


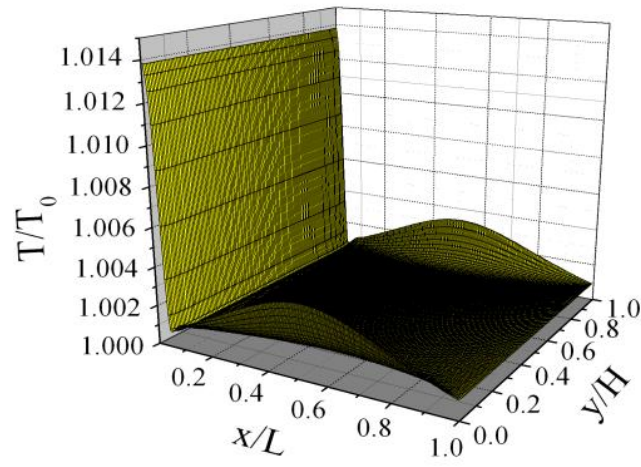

(a)

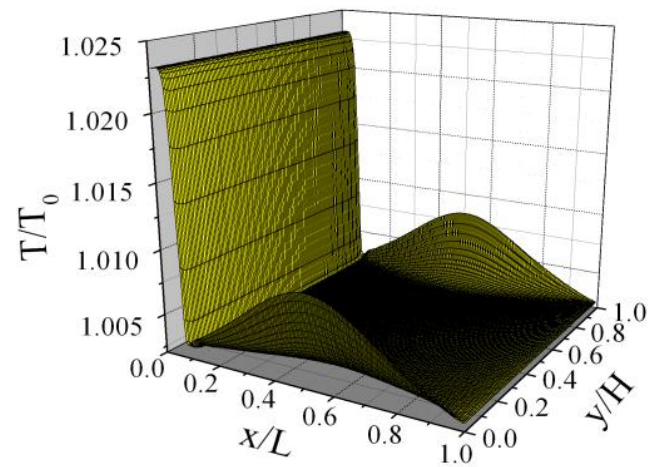

(b)

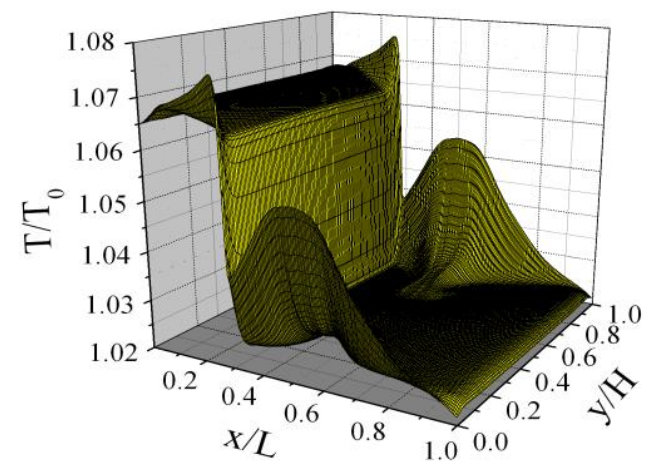

(c)

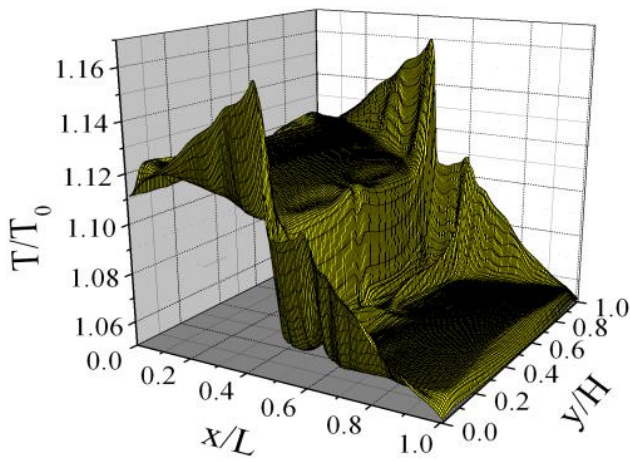

(d)

Figure 5. Instantaneous temperature distributions for four different driving amplitudes at $t c_{0} / 2 L=80$ with the condition of adiabatic boundary for resonant frequency. (a) $l=60 \mu \mathrm{m}$; (b) $l=120 \mu \mathrm{m}$; (c) $l=510 \mu \mathrm{m}$; (d) $l=1040 \mu \mathrm{m}$

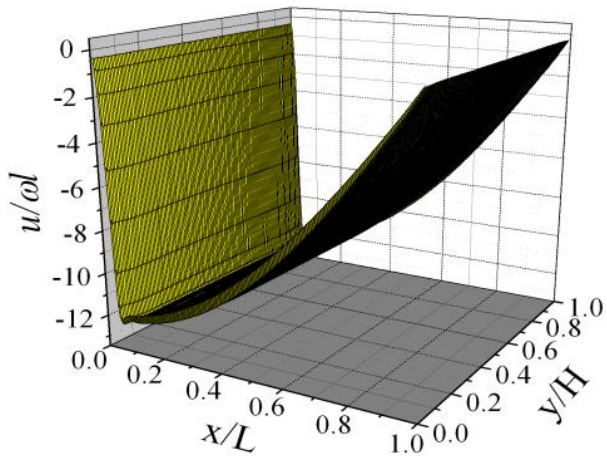

(a)

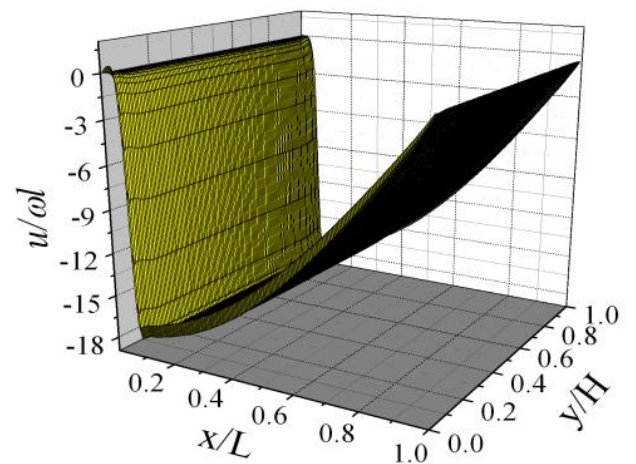

(b)

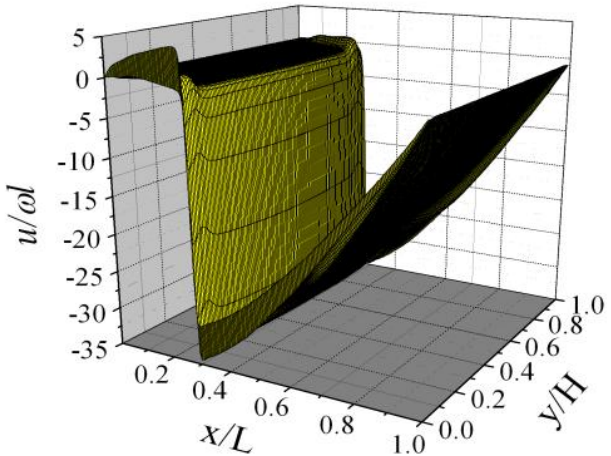

(c)

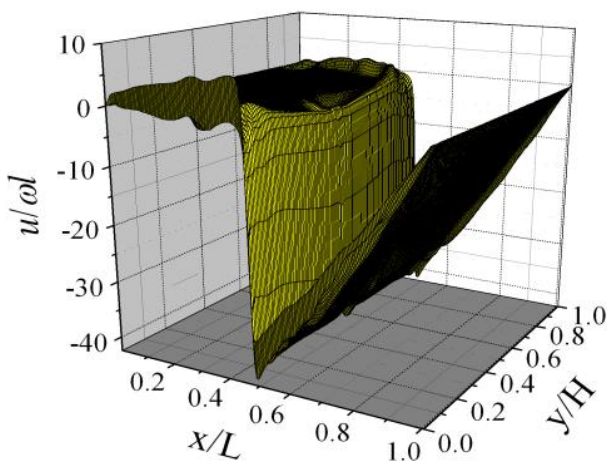

(d)

Figure 6. Instantaneous axial velocity $u$ distributions for four different driving amplitudes at $t c_{0} / 2 L=80$ with the condition of adiabatic boundary for resonant frequency. (a) $l=60 \mu \mathrm{m}$; (b) $l=120 \mu \mathrm{m}$; (c) $l=510 \mu \mathrm{m}$; (d) $l=1040 \mu \mathrm{m}$ 


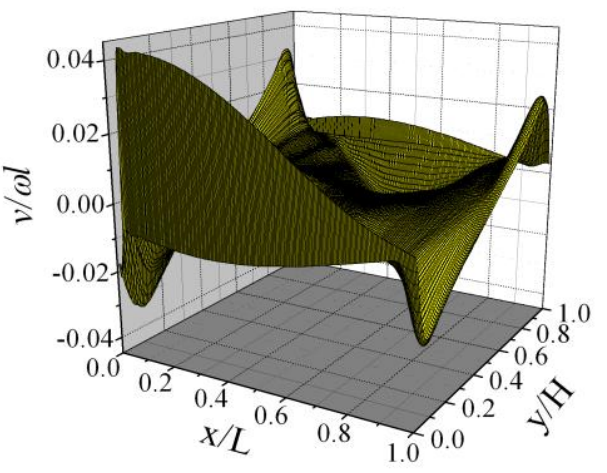

(a)

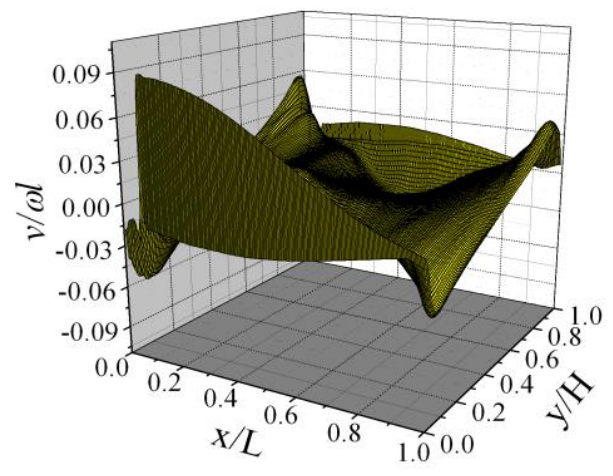

(b)

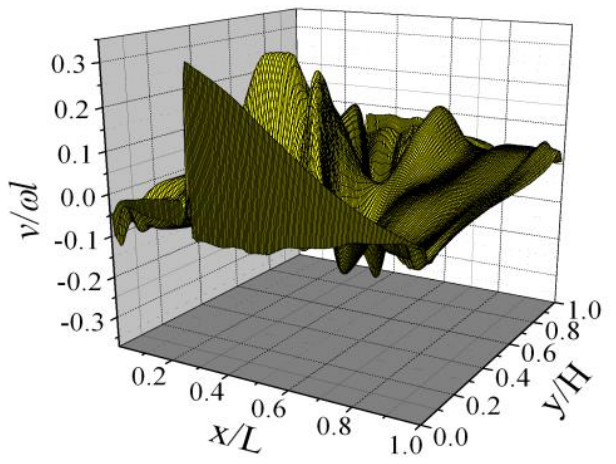

(c)

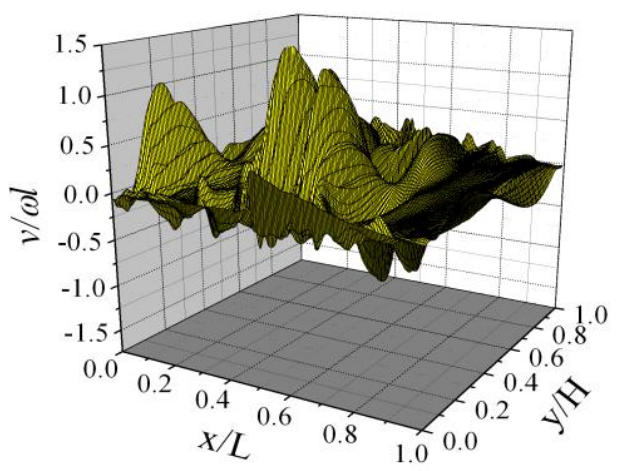

(d)

Figure 7. Instantaneous radial velocity $u$ distributions for four different driving amplitudes at $t c_{0} / 2 L=80$ with the condition of adiabatic boundary for resonant frequency. (a) $l=60 \mu \mathrm{m}$; (b) $l=120 \mu \mathrm{m}$; (c) $l=510 \mu \mathrm{m}$; (d) $l=1040 \mu \mathrm{m}$

Figs. 4-7 show that the nonlinearity has a greater effect on the acoustic field in the resonator with an increase in the driving amplitude. In particular, the distributions of the pressure, temperature and velocity become more complex in large amplitude cases $(l=510 \mu \mathrm{m}$ and $1040 \mu \mathrm{m})$, which are completely different from the finite amplitude cases $(l=60 \mu \mathrm{m}$ and $120 \mu \mathrm{m})$. Then, the distribution of the first-order through the third-order harmonic were investigated at the closed end to analyze the reason for these nonlinear phenomena.

Table 2 lists the relative amplitude of the some orders of harmonic in the pressure wave at the closed end of the resonator driven by four different amplitudes. Fig. 8 shows the Fourier transform of the pressure signal at the closed end of the resonator for four different cases, where $p_{a}$ is the acoustic pressure; that is, $p_{a}=p-p_{0}$. Fig. 4 shows that the amplitudes of all harmonics increase with an increase in the driving amplitude and that the ratio of the second-order harmonic to the fundamental wave varies a little when the driving amplitude $l \leq 510 \mu \mathrm{m}$, which shows that the nonlinear dissipation increases with an increase in the driving amplitude. However, for the driving amplitude $l=1040 \mu \mathrm{m}$, there is not an evident increase in the fundamental wave compared with the case of $l=510 \mu \mathrm{m}$, and there are decreases for the amplitude of the second and third order harmonics. However, it is not the fact that there more nonlinear dissipation for smaller amplitude $l=510 \mu \mathrm{m}$. The reasons for the decrease can be seen in Figs.8(c) and 8(d). The amplitudes of the second- and third-order harmonics for the driving amplitude $l=1040 \mu \mathrm{m}$ are smaller than those for $l=$ $510 \mu \mathrm{mm}$, but the bandwidth of the former is larger than that of the latter, which shows the qualifying factor is lower for $l=$ $1040 \mu \mathrm{m}$. In fact, Fig.8 shows that the bandwidth increases with the driving amplitude, which implies that the qualifying factor becomes larger and larger and the nonlinear dissipation increases more and more with an increase in the driving amplitude.

Table 2. Harmonics at the closed end of resonator driven by four different amplitudes

\begin{tabular}{lccccc}
\hline Case & $p_{1} / p_{0}$ & $p_{2} / p_{0}$ & $p_{3} / p_{0}$ & $p_{2} / p_{1}$ & $p_{3} / p_{1}$ \\
\hline$l=60 \mu \mathrm{m}$ & 0.0292 & 0.0102 & 0.0069 & $34.93 \%$ & $23.63 \%$ \\
$l=120 \mu \mathrm{m}$ & 0.0458 & 0.0149 & 0.0102 & $32.53 \%$ & $22.27 \%$ \\
$l=510 \mu \mathrm{m}$ & 0.1057 & 0.0358 & 0.0181 & $33.87 \%$ & $17.12 \%$ \\
$l=1040 \mu \mathrm{m}$ & 0.1058 & 0.0258 & 0.0135 & $24.39 \%$ & $12.76 \%$ \\
\hline
\end{tabular}

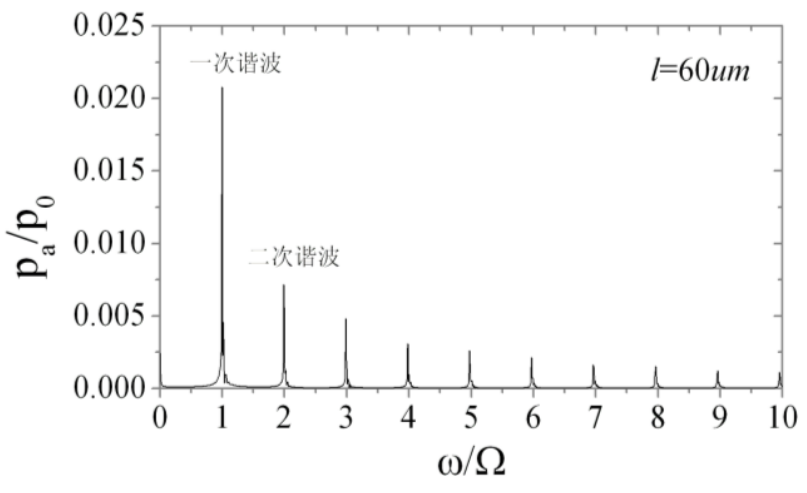

(a) 


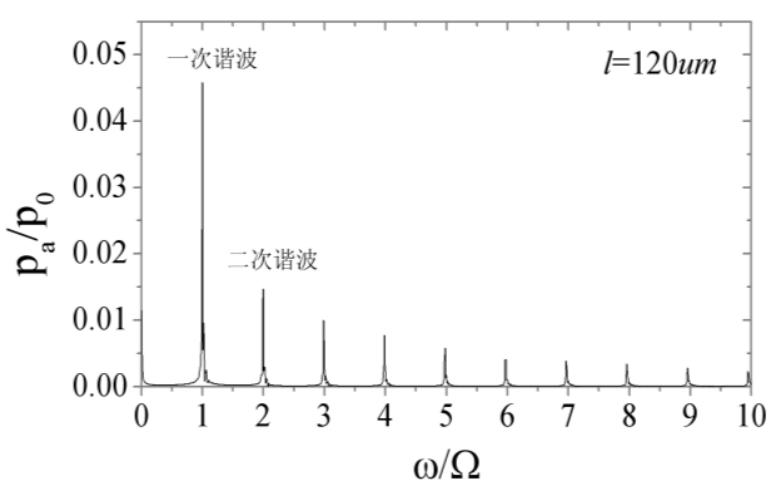

(b)

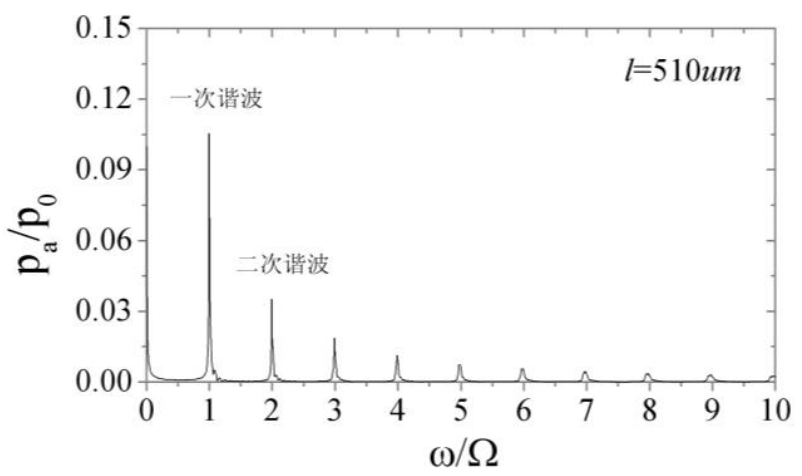

(c)

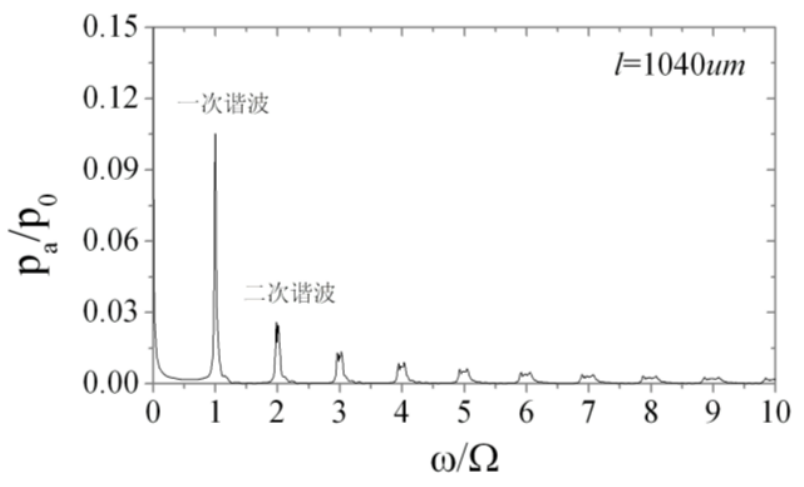

(d)

Figure 8. FFT of the pressure signals under four different driving-amplitudes

\section{CONCLUSION}

In this paper, a gas-kinetic scheme is used to simulate the flow field in a resonator driven by four different driving amplitudes covering finite and large amplitudes. Through the simulation results, the effects of driving amplitude on the acoustic field are investigated. Moreover, the reasons for some nonlinear effects are analyzed. Some significant facts have been revealed. 1) With an increase in the driving amplitude, the waveforms of all acoustics variables are more greatly distorted. When the driving amplitude varies from finite amplitude to large amplitude, the temperature and the axial velocity transform from quasi-one-dimension to twodimensions and the radial velocity transforms from regular to irregular. Moreover, the ratio of radial velocity to axial velocity is negligible for finite amplitude driving, while it is significantly larger in the case of large amplitude. This all indicates that it is necessary to use a multidimensional model to depict the large amplitude oscillation in the resonator. 2) All nonlinear effects on the acoustic field in the resonator become more and more significant with an increase in driving amplitude. The acoustic field under a large amplitude is completely different from that under finite amplitude. In the case of large amplitude, there are annular effects in velocity and temperature profiles both before and after shock waves are observed. Moreover, the reverse velocity phenomena are observedin the region after shock waves in the vicinities of the upper and lower solid walls. While in the case of finite amplitude, no annular effect or reverse velocity phenomenon is observed in the velocity field, and annular effects in the temperature profile are only observed in the region before shock wave. 3) The shock wave has a great effect on pressure, temperature and axial velocity, and has little effect on radial velocity. 4) The nonlinear dissipations increase with an increase in driving amplitude. The qualifying factors of the pressure wave become smaller with an increase in driving amplitude.

\section{ACKNOWLEDGMENT}

This work was supported by the National Natural Science Foundation of China (Nos. 51405157 and 51476052) and the Natural Science Foundation of Hunan (No. 2015JJ3065).

\section{REFERENCES}

[1] M. Nabavi, K. Siddiqui and J. Dargahi, "A fourthorder accurate scheme for solving highly nonlinear standing wave equation in different thermoviscous fluids," J. Comp. Acous., vol. 16, no. 4,pp. 563-576, 2008. DOI: $10.1142 / \mathrm{S} 0218396$ X08003786.

[2] F. L. Ning and X. F. Li, "Numerical simulation of finite amplitude standing waves in acoustic resonators using finite volume method," Wave Motion, vol. 50, no.
2

10.1016/j.wavemoti.2012.08.001.

[3] Y. A. Ilinskii, B. Lipkens, T. S. Lucas, et al., "Nonlinear standing waves in an acoustical resonator," J. Acoust. Soc. Am., vol. 104, no. 5, pp.2664-2674, 1998. DOI: $\underline{10.1121 / 1.423850}$.

[4] Y. D. Chun and Y. H. Kim, "Numerical analysis for nonlinear resonant oscillations of gas in axisymmetric closed tubes," J. Acoust. Soc. Am., vol. 108, no. 6, pp. 2765-2774, 2000. DOI: 10.1121/1.1312363.

[5] C. Vanhille and C. Campos Pozeulo, "Numerical model for nonlinear standing waves and weak shocks in thermoviscous fluids," J. Acoust. Soc. Am., vol. 109, no. 6, pp.2660-2667, 2001. DOI: 10.1121/1.1366318.

[6] W. Chester, "Resonant oscillations in closed tube," J FLUID. MECH., vol. 18, no. 1, pp, 44-64, 1964. DOI: $10.1017 / \mathrm{S} 0022112064000040$.

[7] C. Vanhille and C. Campos-Pozuelob, "A high-order finite-difference algorithm for the analysis of standing acoustic waves of finite but moderate amplitude," $J$ COMPUT. PHYS., vol. 165, no. 2, pp. 334-353, 2000. DOI: $10.1006 / j \mathrm{cph} .2000 .6611$.

[8] C. Vanhille and C. Campos-Pozeulo, "Numerical simulation of two-dimensional nonlinear standing 
acoustic waves," J. Acoust. Soc. Am., vol. 116 no. 1, pp. 194-200, 2004. DOI: 10.1121/1.1760798.

[9] C. Vanhille and C. Campos-Pozuelo, "Three timedomain computational models for quasi-standing nonlinear acoustic waves, including heat production," J. Comp. Acous., vol. 14, no. 2, pp. 143156, 2006. DOI: 10.1142/S0218396X06002986.

[10] C. Vanhille and C. Campos-Pozuelo, "Numerical and experimental analysis of strongly nonlinear standing acoustic waves in axisymmetric cavities," Ultrasonics, vol. 43, no. 8, pp. 652-660, 2005. DOI: $\underline{10.1016 / j . u l t r a s .2005 .02 .001 .}$.

[11] M. Cervenka and M. Bednarik, "Nonlinear standing wave in 2D acoustic resonators," Ultrasonics, vol.44:, no. suppl. pp. e773-e776, 2006. DOI: 10.1016/j.ultras.2006.05.008.

[12] A. Alexeev and C. Gutfingera, "Resonance gas oscillations in closed tubes: Numerical study and experiments," Phys. Fluids, vol. 15, no. 11, pp. $3397-$ 3408, 2003. DOI: 10.1063/1.1613645.

[13] H. Z. Tang, P. Cheng and K. Xu, "Numerical simulations of resonant oscillations in a tube," Numer. Heat Transfer, Part A, vol. 40, no. 1, pp. 3754, 2001. DOI: 10.1080/10407780117498.

[14] Y. Wang, Y. L. He, J. Huang, et al., "Implicit-explicit finite difference lattice Boltzmann method with viscid compressible model for gas oscillating patterns in a resonator," Int. J. Numer. Methods Fluids, vol. 59, no. 8, pp. 853-872, 2009. DOI: 10.1002/fld.1843.

[15] F. Ning and K. Wang, "Two-dimensional model and numerical method for nonlinear standing waves in axisymmetric resonators," presented at 2010 International Conference on Computer, Mechatronics, Control and Electronic Engineering, vol. 5, pp. 68-71. DOI: 10.1109/CMCE.2010.5610033.

[16] A. A. Aganin, M. A.Ilgamov and E. T.Smirnova, "Development of longitudinal gas oscillation in a closed tubes," J SOUND VIB., vol. 195, no. 3, pp. 359-374, 1996. DOI: $10.1006 /$ jsvi.1996.0431.

[17] K. Xu, "Gas-Kinetic Schemes for Unsteady Compressible Flow Simulations," VKI Report, 199803, Jan. 1998.

[18] K. Xu, "A gas-kinetic BGK scheme for the navierstokes equations and its connection with artificial dissipation and Godunov Method," J COMPUT. PHYS, vol. 171, no. 1, pp. 289-335, 2001. DOI: $\underline{10.1006 / \mathrm{jcph} .2001 .6790 .}$.

[19] Zhang Xiaoqing, Feng Heying, Peng Yehui, et al., "Gas-kinetic scheme simulation of gas acoustic oscillating behaviors in a tube," Chinese $J$ ENG THERMOPHYS, vol. 32, no. 3, pp. 403-406, 2011. Available: http://jetp.iet.cn/CN/Y2011/V32/I3/403

[20] Feng H. Y., Zhang X. Q., Peng Y. H., et al., "Numerical simulation of nonlinear acoustic streaming in a resonator using gas-kinetic scheme," J. Appl. Phys., vol. 112, no. 8, pp. 083501, 2012. DOI: $10.1063 / 1.4759345$.

[21] R. A. Saenger and G. E. Hudson, "Periodic shock waves in resonating gas columns," J. Acoust. Soc. Am., vol. 32, no. 8, pp. 961-971, 1960. DOI: $10.1121 / 1.1908343$.

\section{NOMENCLATURE}

$f$

\section{g}

$(x, y)$

$(u, v)$

$(\mathrm{U}, \mathrm{V})$

$\mathrm{T}$

$\mathrm{K}$

W

$\mathrm{S}$

$\mathrm{p}$

e

$\mathrm{F}$

1

L

$\mathrm{H}$

Greek symbols
$\xi$
$\tau$
$\rho$
$\lambda$
$\gamma$
$\omega$

\section{Subscripts}

$\mathrm{x}, \mathrm{y}, \mathrm{t}$ gas distribution function

equilibrium state

Space coordinate

particle velocities

macroscopic velocity

temperature

total number of degrees of freedom macroscopic value

area

pressure

energy

flux

driving amplitude

the length of the resonator

width of the resonator

internal variable

particle collision time

density

wave length

ratio of the principal specific heat

frequency

partial diffential 